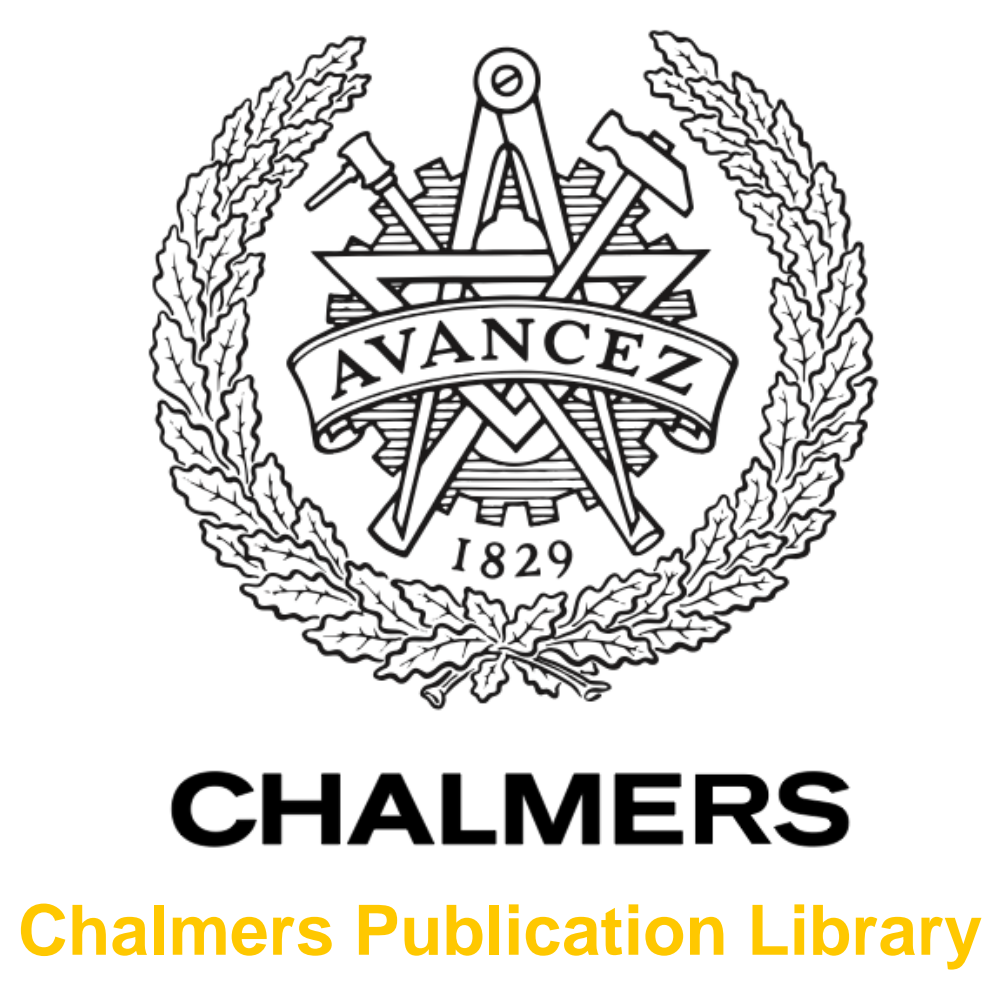

\title{
Proactive control of wind turbine with blade load constraints
}

This document has been downloaded from Chalmers Publication Library (CPL). It is the author's version of a work that was accepted for publication in:

Proceedings of the Institution of mechanical engineers. Part I, journal of systems and control engineering (ISSN: 0959-6518)

Citation for the published paper:

Stotsky, A. ; Egardt, B. (2012) "Proactive control of wind turbine with blade load constraints". Proceedings of the Institution of mechanical engineers. Part I, journal of systems and control engineering, vol. 226(7), pp. 985-993.

http://dx.doi.org/10.1177/0959651812439976

Downloaded from: http://publications.lib.chalmers.se/publication/165122

Notice: Changes introduced as a result of publishing processes such as copy-editing and formatting may not be reflected in this document. For a definitive version of this work, please refer to the published source. Please note that access to the published version might require a subscription. 


\title{
Proactive control of wind turbine with blade load constraints
}

Proc IMechE Part I:

J Systems and Control Engineering 226(7) 985-993

(c) IMechE 2012

Reprints and permissions:

sagepub.co.uk/journalsPermissions.nav DOI: I0.II77/095965।8I2439976

pii.sagepub.com

(SAGE

\section{Alexander Stotsky and Bo Egardt}

\begin{abstract}
This paper describes an easy-to-implement, proactive control strategy for wind turbines, incorporating constraints on blade loads. The control strategy is aimed for rejecting wind gusts and is based on upwind speed measurements and a new statistical wind gust detection mechanism. The control action comprises simultaneous driveline and collective pitch control with constraints on flapwise bending moment. The controller is evaluated by simulation on a transient between two steady-state operational modes of the wind turbine. A new driveline backstepping-based controller with integral action for compensation of steady-state errors is also proposed and verified by simulations.
\end{abstract}

\section{Keywords}

Wind turbine, preview control, collective pitch, load mitigation

Date received: 9 September 20I I; accepted: 30 January 2012

\section{Introduction}

New laser sensor technologies are capable of measuring wind speed at a distance in front of the wind turbine with relatively high sample rates. ${ }^{1-3}$ This allows detection of rapidly changing wind gusts and opens up new opportunities for the development of forward-looking turbine control systems. Model predictive control (MPC) is one of the most suitable proactive techniques based on upwind speed measurements. $^{3-7}$ MPC can successfully cope with rapid transients of the wind speed detected at a distance in front of the turbine. Running MPC is meaningless and computationally expensive in the case where wind speed changes slowly. This necessitates the development of a proactive turbine control strategy that reacts only on the rapid wind speed transients, detected at a distance in front of the wind turbine. To this end the development of a detection mechanism that detects significant changes (transients) in the upwind speed is required. Describing the wind speed as stochastic fluctuations around a time-varying mean value, a statistical detection mechanism that detects significant changes in the mean value of the wind speed is needed.

Collective pitch control ${ }^{7-9}$ is usually used as a tool to limit a turbine power when the wind speed is above rated speed and the generator torque control signal is saturated. Turbine power limitation may be also required due to:

- ageing and wearing of the turbine components;

- nasty sea states in the case of offshore turbines (a sea state is characterized by the wave height, period and power spectrum - the sea state depends on the wind and swell conditions);

- surface roughness on the blades, arising from turbine icing in cold climate;

- some other cases.

The turbine power limitation methods are unified in this paper via the introduction of constraints on blade loads. In this paper the blade loads are understood to be the steady-state blade root flapwise and edgewise bending moments.

Those constraints on loads are usually not taken into account explicitly at the design stage of MPC, and hence the trade-off between load constraints and power production has not been investigated. Despite the fact that the MPC framework allows inclusion of load constraints, in the form of penalty in performance index, to be minimized, ${ }^{10,11}$ the resulting computational burden of MPC might be quite heavy in this case, diminishing its advantages with respect to simple and easy-to-implement control schemes. This in turn motivates the development of a simple and computationally efficient proactive turbine control strategy where the control goals are directly associated with constraints on blade loads.

Signals and Systems, Chalmers University of Technology, Sweden

\section{Corresponding author:}

Alexander Stotsky, Signals and Systems, Chalmers University of Technology, Gothenburg SE-4I2 96, Sweden.

Email: alexander.stotsky@chalmers.se 
A new method of limitation of a turbine power via constraints on blade loads is one of the main contributions of this paper. This method quantifies a trade-off between power production and blade load mitigation, which in turn increases the lifetime of the turbine, reduces vibrations and implies significant savings compared to existing methods.

A transient between two steady-state operational modes of a turbine due to a wind gust is studied in this paper as an example. A new proactive control strategy is based on:

- a new statistical upwind gust detection mechanism;

- proactive pitch actuation;

- blade load mitigation when the gust comes to the turbine;

- a new simultaneous closed-loop rotor speed and pitch angle control with load constraints to achieve maximum efficiency.

This proactive controller is able to cope with fast wind speed transients detected at a distance in front of the turbine, and pitch actuation is used as fast control to reduce blade loads compared to slow (due to large rotor inertia) rotor speed control.

Moreover, a new driveline control algorithm is also proposed in this paper. The controller is based on a new integral backstepping control design procedure for compensation of the steady-state errors. The controller does not require knowledge of such turbine parameters as inertias, driveline stiffness and damping and others. However, a priori knowledge of the turbine parameters is used in a feedforward part of the controller. This feedforward part can be made adjustable to compensate for steady-state errors estimated by the integral part of the controller. The controller design is based on a nonlinear model driven by the turbine power unlike designs described in previous papers (see for example Sloth et al. ${ }^{12}$ and references therein), where aerodynamic torque is used as an external input making the model linear, which might simplify a design, but ignores an important dependence of the aerodynamic torque from the turbine speed, which in turn might have an impact on performance and even the stability of a control system. Besides, the resulting controller gains of the backstepping controller are not high compared to the gains of robust controllers reported in previous papers, see again Sloth et al. ${ }^{12}$ High controller gains imply undesirably high generator torque to achieve desired actuation and hence higher than needed power consumption.

Notice that the driveline controller proposed in this paper can also be used to control a driveline of automotive vehicles.

The contributions of this paper can be summarized as follows:

- a new proactive turbine control strategy in the presence of constraints on blade loads;
- a new method of a wind gust detection based on a statistical detection of a transient in a mean value of wind speed;

- a new driveline backstepping-based control method.

This paper is divided into two main parts. A wind turbine model and control problem statement are described in the next section. A backstepping-based driveline control and proactive control strategy are explained in the subsequent section. The paper finishes with some concluding remarks.

\section{Turbine model and problem statement}

\section{Turbine model}

Aerodynamic model. A wind turbine converts energy from the wind to the rotor shaft that rotates at a speed $\omega_{r}$. The power of the wind $P_{\text {wind }}=\frac{1}{2} \rho A V^{3}$ depends on the wind speed $V$, the air density $\rho$, and the swept area $A=\pi R^{2}$ with the rotor radius $R$, when assuming uniformity of the wind speed across the rotor swept area. From the available power in the swept area, the power on the rotor $P_{r}$ is determined by the power coefficient $C_{p}(\lambda, \beta)=P_{r} / P_{\text {wind }}$, which in turn depends on the pitch angle of the blades $\beta$ and the tip-speed ratio $\lambda=\omega_{r} R / V($ see Figure 1$)$

$$
P_{r}=P_{\text {wind }} C_{p}(\lambda, \beta)=\frac{A \rho V^{3} C_{p}(\lambda, \beta)}{2}
$$

The aerodynamic torque applied to the rotor is given as

$$
T_{a}=\frac{P_{r}}{\omega_{r}}=\frac{A \rho V^{3} C_{p}(\lambda, \beta)}{2 \omega_{r}}
$$

Drive train model. The drive train model consists of a low-speed shaft rotating with a speed $\omega_{r}$ and a highspeed shaft rotating with a speed $\omega_{g}$, having inertias $J_{r}$ and $J_{g}$ respectively. The shafts are interconnected by a gear with ratio $N$. A torsion stiffness $K_{s}$ together with

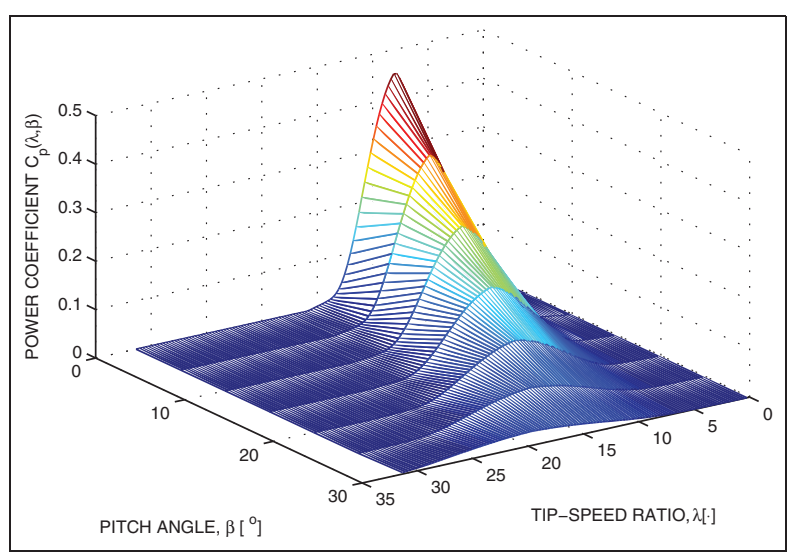

Figure I. Power coefficient $C_{p}(\lambda, \beta)=P_{r} / P_{\text {wind }}$ as a function of the pitch angle of the blades $\beta$ and the tip-speed ratio $\lambda=\omega_{\mathrm{r}} R / V$. 
a torsion damping $K_{d}$ result in a torsion angle $\alpha$ that describes the twist of the flexible shaft.

In summary, the nonlinear model of the drive train with the loading torque from the generator $T_{g}$ as a control action, with the turbine power $P_{r}$ as an input and with rotational speed $\omega_{r}$ as output is given by ${ }^{12,13}$

$$
\begin{aligned}
& J_{r} \dot{\omega}_{r}=\underbrace{\frac{P_{r}}{\omega_{r}}}_{=T_{a}}-K_{s} \alpha-K_{d} \dot{\alpha} \\
& J_{g} \dot{\omega}_{g}=\frac{K_{s}}{N} \alpha+\frac{K_{d}}{N} \dot{\alpha}-T_{g} \\
& \dot{\alpha}=\omega_{r}-\frac{1}{N} \omega_{g}
\end{aligned}
$$

The driveline model (3)-(5) is a nonlinear model driven by the turbine power $P_{r}$ unlike models described in previous papers (see Sloth et al. ${ }^{12}$ ), where aerodynamic torque $T_{a}=P_{r} / \omega_{r}$ is used as an external input making the model linear, which might simplify a design, but does not account for the important dependence of the aerodynamic torque on the turbine speed. Notice that the turbine power also depends on the rotor speed via tipspeed ratio. This dependence can be accounted for via a polynomial model of the power coefficient as a function of tip-speed ratio. This idea is, however, not developed in this paper for the sake of simplicity.

It is assumed that the turbine speed and generator speed are measurable and the torsion angle is estimated using equation (5).

Pitch actuator model. A pitch actuator that tracks a desired command $\beta_{d}$ is modelled as a first-order lag with the rate and range constraints

$$
\begin{aligned}
& \dot{\beta}=-\frac{1}{\tau} \beta+\frac{1}{\tau} \beta_{d}\left(t-t_{d}\right) \\
& |\beta| \leqslant C_{\beta},|\dot{\beta}| \leqslant C_{\dot{\beta}}
\end{aligned}
$$

where $\tau$ is a time constant, $t_{d}$ is a communication delay, and $C_{\beta}$ and $C_{\dot{\beta}}$ are positive constants which define range and rate constraints, respectively.

The parameters of the turbine model described above are detailed in the Appendix.

Steady-state blade operational loads. The loading of wind turbines is extremely complex to model but it is very important for the control design. Steady-state (static) loading is proposed in this paper to describe flapwise and edgewise blade root bending moments on the blades (see IEC-61400-13, Section 3.3.2 'Load Quantities ${ }^{14}$ ). This type of loading is constant (or slowly varying) in time and the resulting deflection of the blades is also constant and proportional to the blade stiffness. Therefore flap- and edgewise blade root bending moments can be described as look-up tables (surfaces in three-dimensional (3D) space) with tipspeed ratio and blade pitch angle as input variables.
Such a surface that describes the flapwise blade root bending moment as a function of tip-speed ratio and blade pitch angle is shown in Figure 3. Notice that the flapwise bending moment depends also on the magnitude of the wind speed, and not only on the tip-speed ratio. This dependence results in a number of sandwiched surfaces of bending moments as a function of absolute value of wind speed, where larger bending moments correspond to higher wind speed. This dependence is not accounted for in this paper for the sake of simplicity. It is assumed that the change of absolute value of wind speed due to a wind gust (a transient from one low wind speed to another one) is small enough and does not have a significant impact on the flapwise bending moment surface.

Notice that static flap- and edgewise bending moments were calibrated via the simulation of a highfidelity computational model of turbine. Stochastic fluctuations in the wind speed model (see the next section) are accounted for via sufficiently long realizations to ensure the statistical reliability/consistency of turbine load estimation. The requirements for the length of these realizations are well formulated in Section 7.5 'Load Calculations' of IEC-61400-1, ${ }^{15}$ and in Section B.2.3 'Mean Load Versus Mean Speed' of IEC-61400-13. ${ }^{14}$

A simple time series wind speed model. The wind is modelled as a discretely measured stochastic process (a sequence of random variables) with a mean value that changes in time and with normally distributed stochastic variations around the mean ${ }^{16}$

$$
V_{k}=V_{m k}+\delta V_{k}
$$

where $E\left[V_{k}\right]=V_{m k}$, where $E$ is a mathematical expectation, and $\delta V_{k}$ is a normally distributed variable with zero mean and variance $\sigma_{k}^{2}$. A wind speed time series is shown in Figure 4.

\section{A turbine power maximization problem statement}

The turbine control problem is to choose a desired generator torque $T_{g}$ and desired blade pitch angle $\beta_{d}$ in order to maximize the turbine power $P_{r}$ under the constraints on the flap- and edgewise bending moments

$$
\begin{aligned}
& P_{r} \rightarrow P_{\text {rmax }} \\
& M_{f}(\lambda, \beta) \leqslant C_{f} \\
& M_{e}(\lambda, \beta) \leqslant C_{e}
\end{aligned}
$$

where $P_{\text {rmax }}$ is the maximum turbine power available under the constraints (10) and (11), and $C_{f}$ and $C_{e}$ are positive constants. Moments $M_{f}(\lambda, \beta)$ and $M_{e}(\lambda, \beta)$ are steady-state flap- and edgewise bending moments respectively.

This problem statement represents a trade-off between turbine power production and blade load mitigation. Tighter constraints on bending moments imply 
a larger drop in power production. In other words, turbine load mitigation is achieved at the expense of turbine power production.

Despite the fact that the steady-state loads are properly accounted for at the design stage of the wind turbine, a blade load control is still required on account of the possible deterioration of the turbine performance due to the temperature variations and ageing and wearing of the turbine components.

\section{Determination of maximum power under load constraints (projection algorithm) and trajectory tracking problem statement}

The next step is to find a desired turbine speed and blade pitch angle that maximize the turbine power under the constraints on the flap- and edgewise bending moments described in the previous section. The maximum power delivered by the turbine is determined by the power coefficient $C_{p}(\lambda, \beta)$ plotted in Figure 1, if the constraints on loads are not considered. The relationship between constraints on load and power production is explained in Figures 2 and 3. In Figure 3 the flapwise bending moment is plotted as a function of tip-speed ratio and pitch angle. Bounding of the flapwise bending moment implies restrictions on the tip-speed ratio and blade pitch angle. Admissible tip-speed ratios and pitch angles are determined by projection of the intersection line (intersection between the flapwise bending moment and its upper bound) plotted in Figure 3 on the plane of tip-speed ratio and pitch angle. Such a projection defines a cut surface in Figure 2 which in turn restricts the turbine power coefficient. The maximum power coefficient under constraints is achieved at the intersection point between the cut surface and the line that defines the maximum coefficient at each value of the

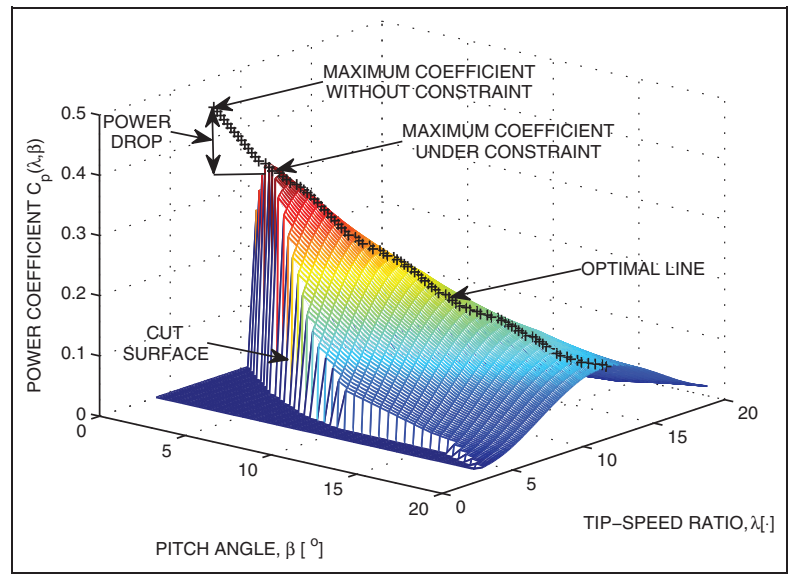

Figure 2. Power coefficient $C_{p}(\lambda, \beta)$ under constraint on the flapwise bending moment. The cut surface that represents the upper bound on the flapwise bending moment restricts the turbine power coefficient. The line that defines a maximum coefficient at each value of the pitch angle is plotted with the black plus signs and is referred to as an optimal line.

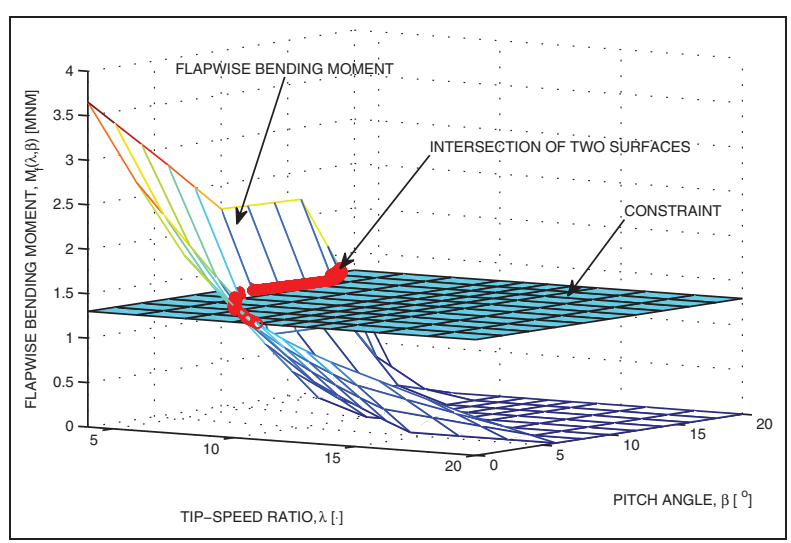

Figure 3. Flapwise bending moment as a function of tip-speed ratio and pitch angle and its upper bound.

pitch angle as shown in Figure 2. Restrictions on the edgewise bending moment can be accounted for in a similar way. Finally, optimal values of the tip-speed ratio and pitch angle which maximize the power coefficient under constraints on flap- and edgewise bending moments can be found. Therefore, the turbine power maximization problem statement described in the previous section is reduced to the tracking problem of the optimal/desired values of the tip-speed ratio and pitch angle.

The turbine control problem is a driveline control problem, namely to choose a desired generator torque $T_{g}$ to track a desired turbine speed $\omega_{r d}$

$$
\lim _{t \rightarrow \infty} \omega_{r}(t)-\omega_{r d}=0
$$

In addition, the blade pitch angle should converge to the desired blade pitch angle $\beta_{d}$

$$
\lim _{t \rightarrow \infty} \beta(t)-\beta_{d}=0
$$

where $\omega_{r d}$ and $\beta_{d}$ are chosen to maximize the power coefficient $C_{p}(\lambda, \beta)$ in the presence of constraints on the blade bending moments (10) and (11).

\section{The driveline and proactive control strategies}

The description of the control strategies is divided into two parts. A driveline backstepping-based control is described in the first part, and a preview-based control strategy for blade load mitigation is explained subsequently. The behaviour of the proactive control strategy is illustrated in an example with a transient between two steady-state operational modes of the turbine caused by a wind gust.

\section{Driveline control based on integral backstepping}

The driveline is controlled via a cascade control (see for example Krstic et al. ${ }^{17}$ and Stotsky et al. ${ }^{18}$ and the references therein) of the driveline torsion angle. The control 
aim is to choose a generator torque $T_{g}$ so as to drive the rotor speed $\omega_{r}$ to a desired constant rotor speed $\omega_{r d}$. Define desired torsion angle $\alpha_{d}$, desired generator speed $\omega_{g d}$ and generator torque $T_{g}$ as

$$
\begin{aligned}
& \alpha_{d}=\underbrace{\frac{\widehat{P_{r}}}{\omega_{r d} K_{s}}}_{\text {feedforward part }}+\underbrace{\gamma_{r} \tilde{\omega}_{r}+\gamma_{r 1} \tilde{\omega}_{r 1}}_{\text {feedback part }} \\
& \tilde{\omega}_{r}=\omega_{r}-\omega_{r d} \\
& \dot{\tilde{\omega}}_{r 1}=\tilde{\omega}_{r} \\
& \omega_{g d}=\underbrace{N \omega_{r d}}_{\text {feedforward part }}+\underbrace{N \gamma_{\alpha} \tilde{\alpha}+N \gamma_{\alpha 1} \tilde{\alpha}_{1}}_{\text {feedback part }} \\
& \tilde{\alpha}^{=}=\alpha-\alpha_{d} \underbrace{\widehat{\rho_{r}}}_{\text {feedforward part }}+\underbrace{\gamma_{g} \tilde{\omega}_{g}+\gamma_{g 1} \tilde{\omega}_{g 1}}_{\text {feedback part }} \\
& \dot{\tilde{\alpha}}_{1}=\tilde{\alpha}{ }_{\tilde{\omega}_{g} N}=\omega_{g}-\omega_{g d} \\
& \dot{\tilde{\omega}}_{g 1}=\tilde{\omega}_{g}
\end{aligned}
$$

where $\widehat{P_{r}} /\left(\omega_{r d} K_{s}\right)$ and $\widehat{P_{r}} /\left(\omega_{r d} N\right)$ are estimates of the feedforward parts $P_{r} /\left(\omega_{r d} K_{s}\right)$ and $P_{r} /\left(\omega_{r d} N\right)$ respectively and $\gamma_{r}, \gamma_{r 1}, \gamma_{\alpha}, \gamma_{\alpha 1}, \gamma_{g}$ and $\gamma_{g 1}$ are positive gains.

The feedforward parts of the controller (14)-(16) are calculated when equating all the derivatives of the model equations (3) to (5) to zero

$$
\begin{aligned}
& 0=\frac{P_{r}}{\omega_{r d}}-K_{s} \alpha_{f} \\
& 0=\frac{K_{s}}{N} \alpha_{f}-T_{g f} \\
& 0=\omega_{r d}-\frac{1}{N} \omega_{g f}
\end{aligned}
$$

and resolving equations (17) to (19) with respect to feedforward torsion angle $\alpha_{f}=P_{r} /\left(\omega_{r d} K_{s}\right)$, generator speed $\omega_{g f}=N \omega_{r d}$ and generator torque $T_{g f}=P_{r} /\left(\omega_{r d} N\right)$.

The feedforward generator torque $T_{g f}=P_{r} /\left(\omega_{r d} N\right)$ also defines a simple feedforward driveline controller which is similar to the feedforward controller described by Pao and Johnson ${ }^{7}$. A closed-loop system with such a controller shows a robust performance, but a relatively slow convergence. The convergence rate of feedforward controller is improved in this paper via the introduction of feedback loops in equations (14) to (16).

Equations (14) to (16) show that the mismatch between the rotor speed $\omega_{r}$ and the desired rotor speed $\omega_{r d}$ is controlled via the torsion angle $\alpha$ which in turn is controlled via generator speed $\omega_{g}$, and finally the generator torque controls the generator speed. Notice that $\omega_{r}$ can be controlled more effectively via $\alpha$ than via $\omega_{g}$ since $K_{s} \gg>\left(K_{d} / N\right)$.

The main idea behind the rotor speed controller becomes clear when equating $\alpha$ to $\alpha_{d}$ and $\omega_{g}$ to $\omega_{g d}$ with $\gamma_{\alpha 1}=0$ which in turn results in the following stable closed-loop dynamics

$$
\begin{aligned}
& J_{r} \dot{\omega}_{r}=-\frac{P_{r} \tilde{\omega}_{r}}{\omega_{r} \omega_{r d}}-\left(\gamma_{r} K_{s}+K_{d}\right) \tilde{\omega}_{r}-\gamma_{r 1} K_{s} \tilde{\omega}_{r 1} \\
& \dot{\tilde{\omega}}_{r 1}=\tilde{\omega}_{r}, \omega_{r}(0)=\omega_{r 0}>0
\end{aligned}
$$

from which it is clear that the control aim (12) is reached. The closed-loop performance is regulated via positive coefficients $\gamma_{r}$ and $\gamma_{r 1}$.

Combining equations (3) to (5) and (14) to (16) results in the following error model

$$
\begin{aligned}
& \dot{\tilde{\omega}}_{g}=-a_{g}^{g} \tilde{\omega}_{g}+a_{\alpha}^{g} \tilde{\alpha}+a_{\omega r}^{g} \tilde{\omega}_{r}+S_{g} \\
& \dot{S}_{g}=-a_{g 1}^{g} \tilde{\omega}_{g}+a_{\alpha 1}^{g} \tilde{\alpha}+a_{\omega r 1}^{g} \tilde{\omega}_{r} \\
& \dot{\tilde{\alpha}}=-a_{\alpha}^{\alpha} \tilde{\alpha}+a_{w r}^{\alpha} \tilde{\omega}_{r}+a_{w g}^{\alpha} \tilde{\omega}_{g}+S_{\alpha} \\
& \dot{S}_{\alpha}=-a_{\alpha 1}^{\alpha} \tilde{\alpha}+a_{\omega r 1}^{\alpha} \tilde{\omega}_{r} \\
& \dot{\tilde{\omega}}_{r}=-a_{w r}^{w r} \tilde{\omega}_{r}+a_{w g}^{w r} \tilde{\omega}_{g}+a_{\alpha}^{w r} \tilde{\alpha}+S_{r} \\
& \dot{S}_{r}=-a_{w r 1}^{w r} \tilde{\omega}_{r}+a_{\alpha 1}^{w r} \tilde{\alpha}
\end{aligned}
$$

where $a_{\alpha}^{g}, a_{\omega r}^{g}, a_{\alpha 1}^{g}, a_{\omega r 1}^{g}, a_{w r}^{\alpha}, a_{w g}^{\alpha}, a_{\omega r 1}^{\alpha}, a_{w g}^{w r}, a_{\alpha}^{w r}$ and $a_{\alpha 1}^{w r}$ are the coefficients and $a_{g}^{g}, a_{g 1}^{g}, a_{\alpha}^{\alpha}, a_{\alpha 1}^{\alpha}, a_{w r}^{w r}$ and $a_{w r 1}^{w r}$ are positive coefficients, and

$$
\begin{aligned}
& S_{g}=\Delta_{g}-\hat{\Delta}_{g} \\
& S_{\alpha}=\Delta_{\alpha}-\hat{\Delta}_{\alpha} \\
& S_{r}=\Delta_{r}-\hat{\Delta}_{r} \\
& \hat{\Delta}_{g}=a_{g 1}^{g} \tilde{\omega}_{g 1}-a_{\alpha 1}^{g} \tilde{\alpha}_{1}-a_{\omega r 1}^{g} \tilde{\omega}_{r 1} \\
& \hat{\Delta}_{\alpha}=a_{\alpha 1}^{\alpha} \tilde{\alpha}_{1}-a_{\omega r 1}^{\alpha} \tilde{\omega}_{r 1} \\
& \hat{\Delta}_{r}=a_{w r 1}^{w r} \tilde{\omega}_{r 1}-a_{\alpha 1}^{w r} \tilde{\alpha}_{1}
\end{aligned}
$$

where $\Delta_{g}, \Delta_{\alpha}$ and $\Delta_{r}$ are constant unmeasurable disturbances coming from the mismatch between feedforward parts $P_{r} /\left(\omega_{r d} K_{s}\right)$ and $P_{r} /\left(\omega_{r d} N\right)$ and their estimates $\widehat{P_{r}} /\left(\omega_{r d} K_{s}\right)$ and $\widehat{P_{r}} /\left(\omega_{r d} N\right)$ used in the controller (14)(16). Disturbances $\Delta_{g}, \Delta_{\alpha}$ and $\Delta_{r}$ are estimated via integral parts $\hat{\Delta}_{g}, \hat{\Delta}_{\alpha}$ and $\hat{\Delta}_{r}$ of the controller (14)-(16).

Exponential stability of the system (20)-(31) can be proved using the following Lyapunov function

$$
\begin{aligned}
V= & \frac{1}{2} \tilde{\omega}_{g}^{2}+\frac{1}{2}\left(\tilde{\omega}_{g}-S_{g}\right)^{2}+\frac{1}{2} \tilde{\alpha}^{2}+\frac{1}{2}\left(\tilde{\alpha}-S_{\alpha}\right)^{2} \\
& +\frac{1}{2} \tilde{\omega}_{r}^{2}+\frac{1}{2}\left(\tilde{\omega}_{r}-S_{r}\right)^{2}
\end{aligned}
$$

whose derivative along the solutions of the system is $\dot{V} \leqslant \gamma_{0} V$ where $\gamma_{0}>0$, and hence the following control aims are reached

$$
\begin{aligned}
& \lim _{t \rightarrow \infty} \tilde{\omega}_{r}=0 \\
& \lim _{t \rightarrow \infty} \tilde{\alpha}=0 \\
& \lim _{t \rightarrow \infty} \tilde{\omega}_{g}=0 \\
& \lim _{t \rightarrow \infty} S_{r}=0 \\
& \lim _{t \rightarrow \infty} S_{\alpha}=0
\end{aligned}
$$




$$
\lim _{t \rightarrow \infty} S_{g}=0
$$

The achievement of the control aim (32) shows that the rotor speed $\omega_{r}$ tracks the desired rotor speed $\omega_{r d}$. This tracking is achieved via a proper choice of the desired torsion angle $\alpha_{d}$ defined in equation (14). In other words, the shaft is properly twisted according to $\alpha_{d}$ in order to achieve an acceptable tracking performance of the rotor speed. The actual torsion angle $\alpha$ converges to the desired torsion angle due to the achievement of the control aim (33). The convergence of the torsion angle is achieved due to the proper choice of the desired generator speed $\omega_{g d}$, and the convergence of the generator speed to the desired one follows in turn from the achievement of the control aim (34). This convergence is achieved due to the proper choice of the generator torque according to (16).

The last three aims (35)-(37) are estimation aims which show a capability of the controller to estimate the deviations between nominal and actual feedforward parts due to inclusion of the integral terms in controller (14)-(16). Therefore, the values of feedforward parts $\widehat{P_{r}} /\left(\omega_{r d} K_{s}\right)$ and $\widehat{P_{r}} /\left(\omega_{r d} N\right)$ can be adapted so that the following is achieved: $\hat{\Delta}_{g}=\hat{\Delta}_{\alpha}=\hat{\Delta}_{r}=0$.

\section{Proactive turbine control strategy for load mitigation}

A transient between two steady-state operational modes of the turbine due to a wind gust is chosen for explanation of the proactive turbine control strategy. This control strategy is described as a sequence of the following events: a detection of the upwind speed transient, pitch actuation and a closed-loop load tracking.

First event: hypothesis-based detection of the upwind speed transient. The wind gust detection problem is a problem of detecting a significant (with respect to variations), change of the mean value of the wind speed within the wind speed model framework defined in the earlier section 'A simple time series wind speed model'.

A statistical wind speed transient detection can be formulated in terms of a statistical hypothesis test where a transient is defined as a systematic change of the mean value in the following sequence of wind speed measurements: $V_{1}, V_{2}, \ldots, V_{w}$. The hypothesis that the mean value is constant is taken as a null hypothesis which is tested against the alternative hypothesis that a transient is present in the mean value of the abovementioned sample. It is assumed that the variance does not change during the transient. For the hypothesis test the sample variance is compared to the mean square successive difference.

The statistical detection of the wind speed transient is based on examination of the variable

$$
r=\frac{q^{2}}{s^{2}}
$$

where

$$
\begin{aligned}
q^{2} & =\frac{1}{2(w-1)} \sum_{k=1}^{w-1}\left(V_{k+1}-V_{k}\right)^{2} \\
s^{2} & =\frac{1}{w-1} \sum_{k=1}^{w}\left(V_{k}-\bar{V}\right)^{2}
\end{aligned}
$$

and $\bar{V}$ is the sample mean value. The variable $r$ is approximately normally distributed for a sufficiently large sample size, $w>20$, provided $V_{k}$ is normally distributed. The transient is detected provided the variance (40) is essentially larger than (39). This hypothesis test was described by Hald, ${ }^{19}$ and the fractiles of the distribution of $r=q^{2} / s^{2}$ are given in Hald's Table 13.6. Detection of engine transients based on this test was proposed by Stotsky. ${ }^{20}$

A wind gust is detected if the null hypothesis is rejected in favour of the alternative hypothesis, indicating a transient in the mean value of a given sample set of wind speed measurements. This hypothesis test is performed in a window of a certain size which is moving in time, where the most recent value of the wind speed enters the window and the oldest value is discarded (leaves the window) at each step, updating the sample set of wind speed measurements. The wind gust detection mechanism is illustrated in Figure 4.

Notice that occurrence of a wind gust misdetection event implies an unnecessary reduction of blade load and hence turbine power. Therefore a sufficiently small significance level should be chosen in the algorithm described above to reduce a probability of a wind gust mis-detection event.

Notice also that laser preview measurements of the wind speed are usually provided with a lower rate (as a rule the laser update rates do not exceed $10 \mathrm{~Hz}$, and the most common rate is $1 \mathrm{~Hz}$, although higher sample rates will be available at low cost in the future) than measurement rates of other system variables such as

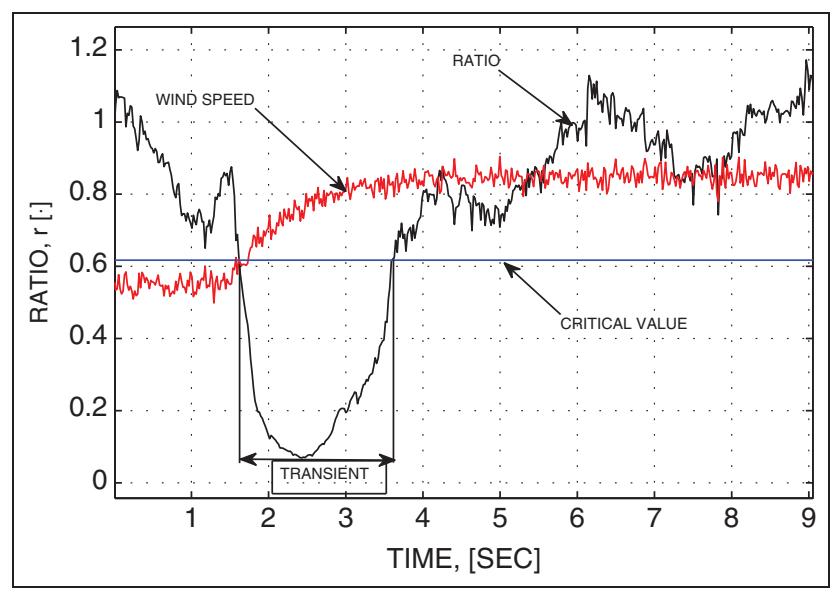

Figure 4. Statistical detection of a wind gust. A wind speed signal in $\mathrm{m} / \mathrm{s}$ is plotted with a red line, with all the values divided by ten. Ratio $r=q^{2} / s^{2}$ defined in equation (38) is plotted with a black line. A wind gust is detected if the ratio $r=q^{2} / s^{2}$ is less than the critical value taken from Table 13.6 of Hald. ${ }^{19}$ 


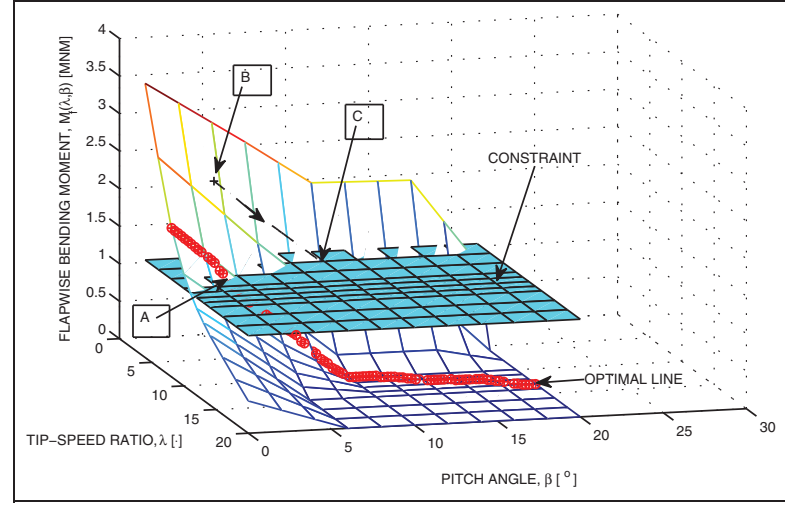

Figure 5. Determination of a desired pitch angle under wind gust. The turbine at steady state is operated at the maximum efficiency point $A$ that satisfies a constraint on a flapwise bending moment. Point $A$ is the meeting point of the optimal line and the constraint surface. The optimal line, plotted with red round signs and plus signs added defines a maximum power coefficient at each value of the pitch angle. An increment of the wind speed due to the wind gust moves the operating point from $A$ to $B$. $A$ desired pitch angle that copes with the wind gust is defined as point $C$.

generator speed, for example, Wang et $\mathrm{al}^{3}$ Wind speed measurements at a sufficiently long distance (commonly around $100 \mathrm{~m}$ ) in front of the turbine allow preprocessing and suitable integration of the laser data to control system, minimizing inaccuracies due to undersampling.

Second event: determination of desired pitch angle for load reduction and pitch actuation. As soon as the wind gust is detected at a distance in front of the turbine, the pitch angle should be increased in order to reduce bending moments on the blades. The algorithm for determination of a desired pitch angle is explained in Figure 5. The turbine at steady state is operated at tip-speed ratio and pitch angle that correspond to the maximum efficiency. This point (point $A$ in Figure 5) is defined as the intersection between the optimal line, in turn defining the maximum power coefficient at each value of the pitch angle, and a constraint surface. An increment of the wind speed implies a reduction of the tip-speed ratio if the turbine speed does not change. The operating point is shifted from point $A$ to point $B$, see Figure 5 , when the wind gust arrives at the turbine. This in turn implies a violation of the constraint on flapwise bending moment. A desired pitch angle that copes with the wind gust is defined from the intersection between the line that corresponds to an expected after-wind-gust tip-speed ratio on the flapwise bending moment surface and the constraint surface, see point $C$ on Figure 5. Such a pitch angle satisfies the constraint on flapwise bending moment when the wind gust arrives at the turbine. The desired pitch angle is sent to the pitch actuator for actuation of the pitch command. The pitch angle values, calculated using the algorithm described

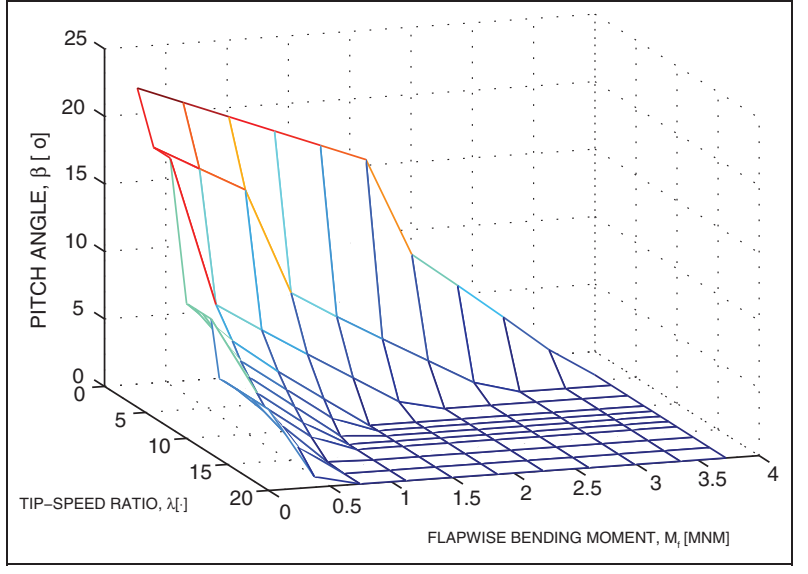

Figure 6. The inverse to the flapwise bending moment look-up table $M_{f}(\lambda, \beta)$. The table gives a pitch angle as a function of tipspeed ratio $\lambda$ and flapwise bending moment $M_{f d}$.

above for a number of wind speeds, can be stored as a look-up table, forming a preview-based feedforward control strategy.

Third event: simultaneous closed-loop speed and pitch angle control for power maximization and load tracking. As soon as the actual pitch angle converges to the desired one the turbine is prepared for load mitigation. When the wind gust has arrived at the turbine and has been successfully alleviated, the rotor speed and pitch angle should be changed as fast as possible to the values that correspond to the maximum power coefficient (point $A$ in Figure 5). A new desired turbine speed is calculated and the driveline control algorithm (14)-(16) described earlier (section 'Driveline control based on integral backstepping') is applied to drive the turbine speed to the desired one. Simultaneously, the pitch angle should be driven to the desired pitch under the constraints on flapwise bending moment. The controller for the pitch angle is based on a look-up table that is inverse to the flapwise bending moment look-up table $M_{f}(\lambda, \beta)$. This inverse look-up table $M_{f}^{-1}\left(\lambda, M_{f d}\right)$ shown in Figure 6 has two inputs: tip-speed ratio $\lambda$ and desired flapwise bending moment $M_{f d}$. A pitch regulator is defined as $\beta_{d k}=M_{f}^{-1}\left(\lambda_{k}, M_{f d}\right)$, where $\lambda_{k}=\omega_{r k} R / V_{k}, V_{k}$ is the wind speed measurements at the turbine site and $\omega_{r k}$ is regulated by the control algorithm (14)-(16). As a desired value of the flapwise bending moment $M_{f d}$, the upper bound $C_{f}$, slightly downshifted to account for variations, can be taken. Hence, the flapwise bending moment is explicitly regulated in a closed loop at this stage, and both rotor speed and pitch angle converge rapidly to the values that correspond to the maximum power coefficient. The convergence of the pitch angle is guaranteed due to the uniqueness of the optimal point on the lambdapitch angle plane. A time chart of all the events described above is shown in Figure 7. 


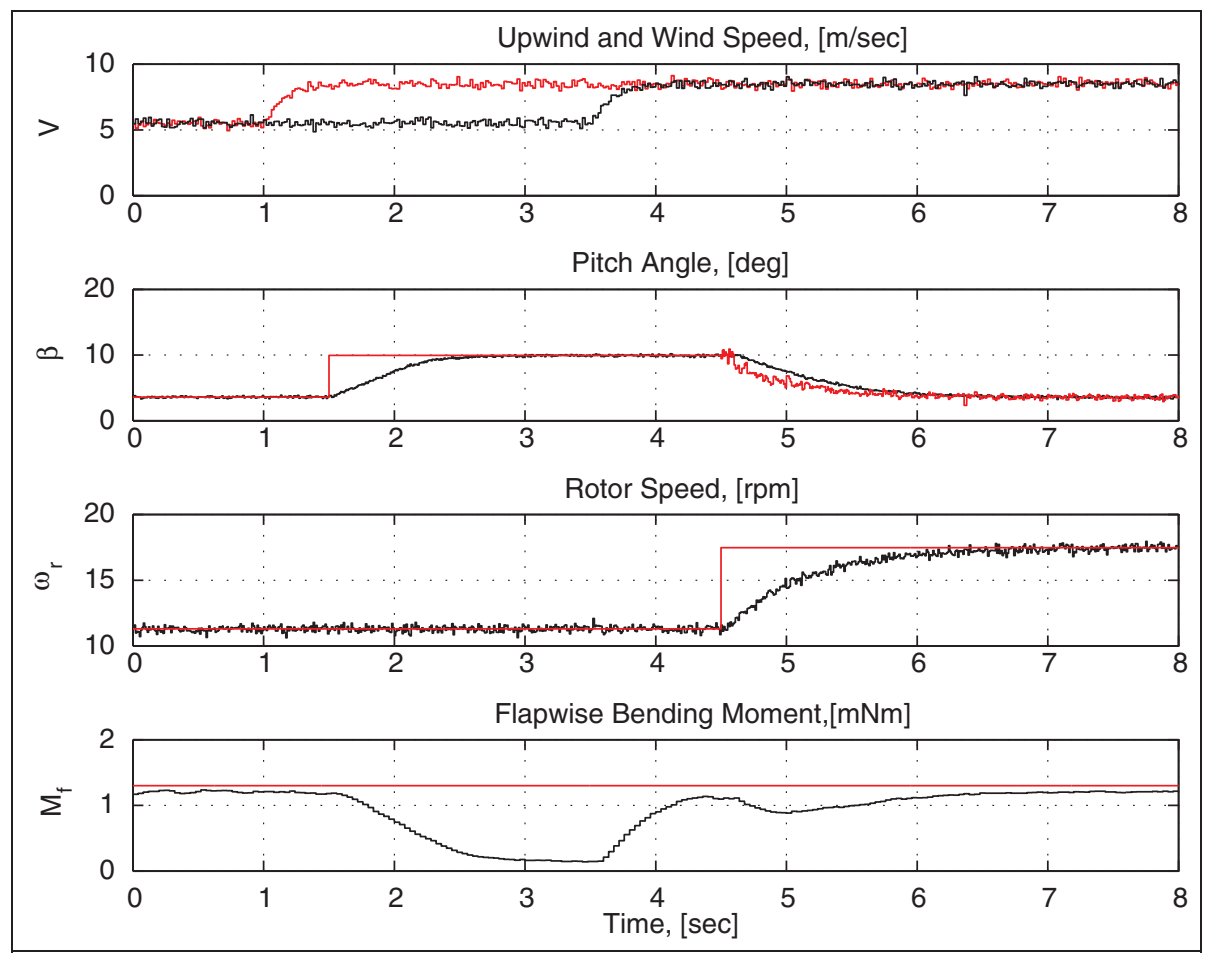

Figure 7. The time chart of upwind/wind speed (red and black lines respectively on the first subplot), desired and actual pitch angles (red and black lines respectively on the second subplot), desired and actual rotor speeds (red and black lines respectively on the third subplot) and flapwise bending moment (black line on the fourth subplot) of forward-looking control strategy. A wind gust arrives at a distance in front of the turbine at $I \mathrm{~s}$ and is detected shortly afterwards using the statistical detection algorithm described in the text (section 'First event: hypothesis-based detection of the upwind speed transient'). A desired pitch angle, calculated according to the algorithm described in the text (section 'Second event: determination of desired pitch angle for load reduction and pitch actuation'), is sent to the pitch actuator at $1.5 \mathrm{~s}$. The actual pitch angle converges to the desired pitch angle before the time when the gust arrives at the turbine (the gust arrives at the turbine at $3.5 \mathrm{~s}$ ). Finally, the rotor speed and pitch angle are regulated simultaneously to the values that correspond to the maximum power coefficient in the presence of constraints, using the algorithm described in the text (section 'Third event: simultaneous closed-loop speed and pitch angle control for power maximization and load tracking') All the regulation is performed without any violation of the constraint on flapwise bending moment plotted with a red line on the fourth subplot.

\section{Conclusion}

A new and easy-to-implement proactive turbine control concept has been described. The concept opens new opportunities for simultaneous turbine and control system design taking into account the constraints on steady-state blade loads. This in turn allows a choice of the optimal turbine operational modes associated with a trade-off between blade load alleviation and turbine power production.

\section{Funding}

This work was supported by the Swedish Wind Power Technology Center (SWPTC).

\section{References}

1. Frehlich R. and Kelley N. Measurements of wind and turbulence profiles with scanning Doppler lidar for wind energy applications. IEEE J Select Topics Appl Earth Observ Remote Sens (J-STARS) 2008; 1: 42-47.

2. Mikkelsen T, Hansen K, Angelou N, et al. Lidar wind speed measurements from a rotating spinner. In:
European wind energy conference and exhibition, Warsaw, Poland, April 2010.

3. Wang N, Johnson $\mathrm{K}$ and Wright A. LIDAR-based FX-RLS feedforward control for wind turbine load mitigation. In: American control conference on O'Farrell Street, San Francisco, 29 June-1 July 2011, pp. 19101915.

4. Bossanyi E, Savini B, Iribas M, et al. Advanced controller research for multi-MW wind turbines in the UPWIND project. Wind Energy, Epub ahead of print 2011. DOI: $10.1002 /$ we.523.

5. Henriksen L and Poulsen N. Model predictive control of a wind turbine with constraints. In: Proceedings of EWEC 2008, Brussels, Belgium, March 2008.

6. Laks J, Pao L, Wright A, et al. The use of preview wind measurements for blade pitch control. Mechatronics 2011; 21: 668-681.

7. Pao L and Johnson K. A tutorial on the dynamics and control of wind turbines and wind farms. In: Proceedings of the American control conference, St. Louis, MO, 10-12 June 2009, pp.2076-2089.

8. Hansen M, Hansen A, et al. Control design for a pitchregulated, variable speed wind turbine. Roskilde, Denmark: Riso National Laboratory, January 2005. 
9. Soleimanzadeh $\mathrm{M}$ and Wisniewski R. Controller design for a wind farm, considering both power and load aspects. Mechatronics 2011; 21: 720-727.

10. Biegel B, Juelsgaard M, Kraning M, et al. Wind turbine pitch optimization. In: IEEE international conference on control applications (CCA), Part of 2011 IEEE multiconference on systems and control, Denver, CO, 28-30 September 2011, pp.1327-1334.

11. Soltani M, Wisniewski R, Brath $\mathrm{P}$, et al. Load reduction of wind turbines using receding horizon control. In: Proceedings of the IEEE international conference on control applications (CCA), Part of 2011 IEEE multi-conference on systems and control Denver, CO, 28-30 September 2011, pp.852-857.

12. Sloth C, Esbensen T, Niss M, et al. Robust LMI-based control of wind turbines with parametric uncertainties. In: Proceedings of the 18th IEEE international conference on control applications, Part of 2009 IEEE multiconference on systems and control St Petersburg, Russia, 8-10 July 2009, pp.776-781.

13. Ostergaard K, Stoustrup J and Brath P. Linear parameter varying control of wind turbines covering both partial load and full load conditions. Int $J$ Robust Nonlin Contr 2008; 19: 92-116.

14. IEC 61400-13 Ed.1. Wind turbine generator systems 13: measurements of mechanical loads. Geneva: International Electrotechnical Commission, 2001-06.

15. IEC 61400-1 Ed.3. Wind turbines 1: design requirements. Geneva: International Electrotechnical Commission, 2005-08.

16. Freris L. Wind energy conversion systems. London: Prentice-Hall, 1990.

17. Krstic M, Kanellakopoulos I and Kokotovich P. Nonlinear and adaptive control design. New York: Wiley, 1995.

18. Stotsky A, Hedrick JK and Yip PP. The use of sliding modes to simplify the backstepping control method. In: Proceedings of American control conference, Albuquerque, NM, 1997, pp.1703-1708.

19. Hald A. Statistical theory with engineering applications. New York: Wiley, 1952.

20. Stotsky A. Automotive engines: control, estimation, statistical detection. Berlin: Springer, 2009.

\section{Bibliography}

Burton T, Sharpe D, Jenkins N and Bossanyi E. Wind energy handbook. New York: Wiley, 2001.

Johnson K, Pao L, Balas M and Fingersh L. Control of variable-speed wind turbines: standard and adaptive techniques for maximizing energy capture. IEEE Contr Syst Mag 2006; 26: 70-81.

Kusiak A, Song Z and Zheng H. Anticipatory control of wind turbines with data-driven predictive models. IEEE Trans Energ Convers 2009; 24: 766-774.

\section{Appendix}

\section{Notation}

\section{Aerodynamic variables}

$C_{p}(\lambda, \beta) \quad$ power coefficient (-)

$M_{e}(\lambda, \beta) \quad$ edgewise bending moment $(\mathrm{N} \mathrm{m})$

$M_{f}(\lambda, \beta) \quad$ flapwise bending moment $(\mathrm{N} \mathrm{m})$

$P_{r} \quad$ rotor power $\left(\mathrm{W}=\mathrm{Kg} \mathrm{m}^{2} / \mathrm{s}^{3}\right)$

$P_{\text {wind }} \quad$ wind power (W)

$T_{a} \quad$ aerodynamic torque $\left(\mathrm{Nm}=\mathrm{Kg} \mathrm{m}^{2} / \mathrm{s}^{2}\right)$

$V \quad$ wind speed $(\mathrm{m} / \mathrm{s})$

$\beta \quad$ blade pitch angle (deg)

$\dot{\beta} \quad$ blade pitch rate $(\mathrm{deg} / \mathrm{s})$

$\lambda \quad$ tip-speed ratio $(-)$

\section{Driveline variables}

$\begin{array}{ll}T_{g} & \text { generator torque }(\mathrm{N} \mathrm{m}) \\ \alpha & \text { torsion angle }(\mathrm{rad}) \\ \dot{\alpha} & \text { torsion rate }(\mathrm{rad} / \mathrm{s}) \\ \omega_{g} & \text { generator speed }(\mathrm{rad} / \mathrm{s}) \\ \omega_{r} & \text { rotor speed }(\mathrm{rad} / \mathrm{s})\end{array}$

\section{Parameters}

A

$J_{g}$

$J_{r}$

$K_{d}$

$K_{s}$

$N$

$R$

$t_{d}$

$\rho$

$\tau$ swept area $\left(5.026510^{3} \mathrm{~m}^{2}\right)$ generator inertia $\left(60 \mathrm{Kg} \mathrm{m}^{2}=60 \mathrm{~N} \mathrm{~m} \mathrm{~s}^{2}\right)$ rotor inertia $\left(910^{6} \mathrm{Kg} \mathrm{m}^{2}\right)$ damping coefficient $\left(2.510^{5} \mathrm{~N} \mathrm{~m} \mathrm{~s} / \mathrm{rad}\right)$ stiffness coefficient $\left(1.610^{8} \mathrm{~N} \mathrm{~m} / \mathrm{rad}\right)$ gear ratio $(83)$ rotor radius $(40 \mathrm{~m})$ pitch actuator time delay (0.01 s) air density $1.269\left(\mathrm{Kg} / \mathrm{m}^{3}\right)$ pitch actuator time constant (0.25 s) 